DOI: https://doi.org/10.34069/RA/2020.6.05

Volumen 3, Número 6/julio-diciembre 2020

\title{
Canavalia ensiformis ( $L$ ): en propiedades químicas de un suelo fluvisol diferenciado
}

\section{Canavalia ensiformis (L): in the chemical properties of a Eutric Differentiated Soils}

\section{Canavalia ensiformis (L): em propriedades químicas de um solo de fluvisol diferenciado}

Recibido: 12 de octubre de $2020 \quad$ Aceptado: 17 de diciembre de 2020

\author{
Autores: \\ Osmara Renté Martí10 \\ Pablo Pablos Reyes ${ }^{11}$ \\ Yeline Corrales Vila ${ }^{12}$ \\ Maritza Cuevas Rodríguez ${ }^{13}$ \\ María Caridad Nápoles García ${ }^{14}$
}

\section{Resumen}

Evaluar el efecto de diferentes manejos de Canavalia ensiformis como cultivo de cobertura (CC) en propiedades químicas de un suelo Fluvisol Diferenciado fue el objetivo del trabajo. Se desarrolló en un área productiva cooperativa, en el municipio Santiago de Cuba, en condiciones de secano. Los tratamientos se establecieron atendiendo a un testigo que representó la cobertura espontánea del suelo (barbecho) y dos tratamientos con cobertura de Canavalia durante 80 días: uno sin incorporar al suelo, otro incorporado y el último como cobertura permanente en el suelo durante 120 días. Se evaluó la biomasa fresca y seca, el carbono de la biomasa (CBIOMASA), el CBIOMASA integrado al COS, la relación de Biomasa - C - CO2, y se estimó el carbono secuestrado y su captura posterior en el CO y COT y contenido de nutrientes. Los resultados fueron positivos en la mejora del suelo con respecto a su condición inicial, cuando la cobertura de Canavalia es incorporada al suelo o es mantenida permanente; el carbono estimado mostro incrementos en el contenido de C orgánico y de los nutrientes como N, P, Ca y Mg en el suelo.

Palabras clave: abonos verdes, leguminosa, secuestro de carbono.

\section{Abstract}

The objective of the study was to evaluate the effect of different Canavalia ensiformis management as a cover crop (CC) on the chemical properties of a differentiated Fluvisol soil. It

\footnotetext{
${ }^{10}$ MSc. Profesora Asistente. Ingeniera Agrónoma. Master en Nutrición de plantas y Biofertilizantes. Departamento de Agronomía. Facultad Ingeniería Química y Agronomía. Universidad de Oriente, UO. Santiago de Cuba. Cuba. https://orcid.org/0000-0003-3178-1017

${ }^{11}$ Dr.C. Investigador Titular. Doctor en Ciencias Agrícolas. Subdirector de Investigación e Innovación Tecnológica. Estación Territorial de Investigaciones de la Caña de Azúcar, ETICA. Palma Soriano. Santiago de Cuba. Cuba. https://orcid.org/0000-0003-1820-0142

${ }^{12}$ Lic. Ingeniera Agropecuaria. Primer Químico. Subdirección Diagnósticos y Análisis Químicos. Estación Territorial de Investigaciones de la Caña de Azúcar, ETICA. Palma Soriano. Santiago de Cuba. Cuba.

${ }^{13}$ Ingeniera Agropecuaria. Químico Analista. Subdirección Diagnósticos y Análisis Químicos. Estación Territorial de Investigaciones de la Caña de Azúcar, ETICA. Palma Soriano. Santiago de Cuba. Cuba.

${ }^{14}$ Dra.C. Investigador Titular. Doctora en Ciencias Biológicas. Departamento de Fisiología y Bioquímica. Instituto Nacional de Ciencias Agrícolas, INCA. Mayabeque. Cuba. https://orcid.org/0000-0003-1413-1717
} 


\section{AMAZÉNAS}

was developed in a cooperative productive area, in the Santiago de Cuba municipality, under dry conditions. The treatments were established by attending a control that represented the spontaneous cover of the soil (fallow) and two treatments with Canavalia cover for 80 days: one without incorporating the soil, another incorporated and the last as permanent cover on the soil for 120 days. The fresh and dry biomass, the carbon of the biomass (CBiomass), the CBiomass integrated to the $\mathrm{SOC}$, the ratio of Biomass - $\mathrm{C}-\mathrm{CO} 2$ were evaluated, and the sequestered carbon and its subsequent capture in $\mathrm{CO}$ and TOC and content of nutrients. The results were positive in the improvement of the soil with respect to its initial condition, when the Canavalia cover is incorporated into the soil or is maintained permanently; the estimated carbon showed increases in the content of organic $\mathrm{C}$ and nutrients such as $\mathrm{N}, \mathrm{P}, \mathrm{Ca}$ and $\mathrm{Mg}$ in the soil.

Key Words: Green manure, leguminous, Kidnapping of carbon.

\section{Resumo}

O objetivo do estudo foi avaliar o efeito de diferentes manejos de Canavalia ensiformis como cobertura vegetal (CC) nas propriedades químicas de um solo Fluvisol diferenciado. Foi desenvolvido em uma área produtiva cooperativa, no município de Santiago de Cuba, em condições de seca. Os tratamentos foram estabelecidos atendendo a uma testemunha que representava a cobertura espontânea do solo (pousio) e dois tratamentos com cobertura de Canavalia por 80 dias: um sem incorporação do solo, outro incorporado e o último como cobertura permanente do solo por 120 dias. A biomassa fresca e seca, o carbono da biomassa (CBIOMASA), o CBIOMASA integrado ao SOC, a relação Biomassa - C - CO2, e o carbono sequestrado e sua posterior captura em $\mathrm{CO}$ e TOC e teor de nutrientes. Os resultados foram positivos na melhoria do solo em relação ao seu estado inicial, quando a cobertura de Canavalia é incorporada ao solo ou mantida de forma permanente; o carbono estimado mostrou acréscimos no conteúdo de C orgânico e de nutrientes como N, P, Ca e Mg no solo.

Palavras-chave: adubos verdes, leguminosas, sequestro de carbono.

\section{Introducción}

La adopción de la agricultura de conservación requiere de una conversión progresiva de todo el sistema de producción (Beuchelt et al. 2015). Esta agricultura reduce la emisión de $\mathrm{CO}_{2}$, ya que retiene una mayor cantidad de carbono orgánico (CO) en el suelo (Dendooven et al. 2012). Algunos autores (Carvalho, Días, \& Melo, 2006) refieren el uso de leguminosas para el aprovechamiento de la producción de fitomasa y de procesos alelopáticos; además de ser utilizados en el contenido de MO y la estructura del suelo (INIA, 2008).

A este conocimiento se añade, que los abonos verdes permiten proteger, recuperar, aportar y mejorar las condiciones biológicas, físicas y nutricionales del suelo (Jiménez et al, 2011), y pueden condicionar modificaciones cualitativas y cuantitativas en el suelo (Sociedad de agricultores de Chile, 2013). En los últimos años se ha ampliado la definición de abonos verdes / cultivos de cobertura (AV/CC) y se cultivan no sólo para ser incorporados, sino que además se siembran para promover la cobertura del suelo y protegerlo de la erosión (García et al, 2017). Diversos son los resultados que muestran mejora en las propiedades químicas en el suelo cuando se emplean rotaciones que incluyen Canavalia ensiformis como abono verde, ya sean inoculados o no (Martín, Rivera, Arias \& Pérez, 2012). Del mismo modo son utilizados en función del secuestro de C, la reducción de la erosión, en la mejora de la estructura del suelo (INIA, 2015); además de favorecer otras condiciones como el incremento del contenido de $\mathrm{C}$ en el suelo a través de la conservación de la humedad del mismo (Nahed et al. 2013; Anaya \& Huber, 2015). 
La Canavalia es una especie adaptada a las condiciones de Cuba debido a su vigoroso crecimiento. Además, realiza aportes de $\mathrm{N}$ atmosférico fijado al sistema suelo planta por vía de la fijación biológica (FBN) recicla cantidades apreciables de P y K (INIA, 2008). Es considerada la mejor leguminosa para enfrentar frecuentes sequías por ser sumamente resistente y se adapta a condiciones de suelos deteriorado (Bunch, 2016), por lo que tolera un amplio rango de texturas y fertilidad del suelo; creciendo bien en suelos altamente lixiviados, así como en suelos ácidos, salinos y en suelos arcillosos húmedos (Carvalho, Días, \& Melo, 2006). Las características de esta leguminosa herbácea muy rústica, anual o bienal y de crecimiento determinado, posee una alta sensibilidad al fotoperiodo y su tasa de crecimiento es rápida. Lo que permite resistir períodos de sequía por su profundo sistema radical, que sobrevive a la humedad almacenada en el suelo (Embrapa, 2007).

El suelo Fluvisol diferenciado en estudio contribuye a la producción agrícola del territorio en la producción hortícola y de viandas, sin embargo, limitaciones como el bajo contenido de materia orgánica, la baja retención de la humedad y el nivel de compactación medio además de estar expuesto a condiciones climáticas adversas (altas temperaturas y sequías prolongadas) y prácticas inadecuadas de manejo, no permiten obtener mayores incrementos productivos y contribuyen a limitar la productividad. Por lo que fue objetivo de este estudio evaluar el efecto de la Canavalia ensiformis como Cultivo de cobertura con diferentes manejos en propiedades químicas de un suelo Fluvisol diferenciado.

\section{Metodología}

El experimento se realizó en un área productiva cooperativa, en el municipio y provincia Santiago de Cuba. El área tiene más de 40 años de explotación con régimen de humedad en secano, sobre un suelo Fluvisol Diferenciado (Hernández et al, 2015). Las labores de preparación del suelo (Aradura-Grada ligera-Cruce-Grada ligera) se realizaron con el uso del implemento ADI 3 y grada ligera. A continuación, se realizó el surcado. Se empleó un diseño de bloques al azar, con cuatro tratamientos (Tabla I) y cuatro réplicas. Las parcelas conformadas por ocho surcos de cinco metros de longitud y seis metros de ancho para un área total de $30 \mathrm{~m}^{2}$. La leguminosa se sembró a una distancia de $0,70 \mathrm{~m}$ entre hileras y $0,25 \mathrm{~m}$ entre plantas. No se aplicaron fertilizantes orgánicos e inorgánicos, biofertilizantes y bioestimulantes, en ninguna etapa del cultivo. Las labores fitotécnicas realizadas fueron una labor de limpia y aporque a los 10 días y otra a los 15 días.

Tabla I.

Descripción de los tratamientos evaluados.

\begin{tabular}{|c|c|c|}
\hline No & Tratamiento & Descripción \\
\hline I & Testigo absoluto $(\mathrm{Cn})$ & Representa el suelo con cobertura espontánea (barbecho) \\
\hline II & $\begin{array}{l}\text { Canavalia como cultivo } \\
\text { cobertura sin incorporar } \\
(\mathrm{CCs} / \mathrm{i})\end{array}$ & $\begin{array}{l}\text { Se estableció la Canavalia como cultivo de cobertura } \\
\text { iniciada la floración ( } 80 \text { días), luego fue cortada y expuesta } \\
\text { sobre la superficie del suelo. }\end{array}$ \\
\hline III & $\begin{array}{l}\text { Canavalia como cultivo } \\
\text { cobertura incorporada al } \\
\text { suelo }(\mathrm{CC} / \mathrm{i}) \text {. }\end{array}$ & $\begin{array}{l}\text { La leguminosa como cobertura permaneció hasta la floración } \\
\text { ( } 80 \text { días), se cortó, se fragmentó y se incorporó la biomasa. }\end{array}$ \\
\hline IV & $\begin{array}{l}\text { Canavalia como cultivo } \\
\text { cobertura permanente } \\
\text { (CC/p). }\end{array}$ & $\begin{array}{l}\text { Cobertura permanentede Canavalia durante } 120 \text { días que } \\
\text { duró el estudio. }\end{array}$ \\
\hline
\end{tabular}

Fuente: Elaboración propia. 


\section{AMAZÉNAS}

\section{Determinaciones realizadas}

Los muestreos de suelos se realizaron con barrena agroquímica en todos los tratamientos, en tres momentos: al inicio del estudio (0 días); a los 80 y 120 días, siguiendo la diagonal de la parcela en zigzag, a la profundidad de $0-20 \mathrm{~cm}$. Las muestras estuvieron compuestas por 30 submuestras de 10 - $15 \mathrm{~g}$ de suelo. Los análisis de suelo se realizaron según el Manual de técnicas analíticas para análisis de suelo (Paneque et al, 2010). El pH fue determinado mediante potenciometría en $\mathrm{KCl}$, con una relación suelo-solución de 1:2,5. El nitrógeno total fue determinado por el método Khejdhal. El contenido de fósforo se determinó a partir de la extracción con ácido sulfúrico $0,1 \mathrm{~N}$, en una relación suelo-solución 1:2,5; durante 3 minutos, según Oniani. El potasio y $\mathrm{Na}$ se determinaron mediante fotometría de llama. Los cationes intercambiables Ca y Mg se valoraron en EDTA, realizando la extracción con NH4Ac 1N, pH-7.

El contenido de carbono orgánico $\mathrm{CO}(\%)$ fue determinado por el método analítico de Walkley-Black en 1934. El COT se obtuvo según metodología propuesta (Riofrío, 2007; Bojórquez et al., 2015): COT= CO x Da x P

Dónde: COT: Carbono orgánico total en suelo por superficie (t ha-1);

CO: Carbono orgánico total (\%);

Da: Densidad aparente del suelo $\left(\mathrm{Mg} \mathrm{m}^{-3}\right)$;

P: Profundidad del suelo (m).

Las características químicas del suelo al inicio (0 días) se presentan en la Tabla II.

Tabla II.

Características químicas del suelo Fluvisol Diferenciado en su estado inicial a la profundidad 0$20 \mathrm{~cm}$.

\begin{tabular}{|c|c|c|c|c|c|c|c|c|}
\hline \multirow[t]{2}{*}{$\underset{(\mathrm{KCl})}{\mathrm{pH}}$} & \multirow[t]{2}{*}{$\begin{array}{l}\text { CO } \\
(\%)\end{array}$} & \multirow[t]{2}{*}{$\begin{array}{c}\text { COT } \\
\left(\mathrm{t} \mathrm{ha}^{-1}\right)\end{array}$} & \multirow[t]{2}{*}{$\begin{array}{l}\mathbf{N} \text { total } \\
(\%)\end{array}$} & $\mathrm{P}_{2} \mathrm{O}_{5}$ & $\mathbf{K}_{2} \mathbf{O}$ & $\underset{(2+)}{\mathbf{M g}}$ & $\mathrm{Ca}^{(2+)}$ & $\mathbf{N a}{ }^{(+)}$ \\
\hline & & & & \multicolumn{2}{|c|}{$\left(\mathrm{mg}^{100 \mathrm{~g}^{-1}}\right)$} & \multicolumn{3}{|c|}{$\left(\operatorname{cmol}_{\mathrm{c}} \mathrm{kg}^{-1}\right)$} \\
\hline 6,7 & 1,0 & 25,2 & 0,097 & 199,8 & 42,52 & 4,5 & 16,50 & 0,46 \\
\hline
\end{tabular}

Fuente: Elaboración propia. Datos de laboratorio ETICA. Palma Soriano.

El aporte de biomasa fresca y seca total de la leguminosa se determinó a partir de los 80 días. Se tomaron para ello cinco plantas completas al azar dentro del área de cálculo (cuatro surcos centrales) de cada parcela. Para determinar la masa fresca se pesaron las plantas recién cortadas y fragmentadas en cada una de sus partes (hojas, tallos y raíces) y se expresó gramos por planta. Posteriormente se colocó el material en estufa a $72^{\circ} \mathrm{C}$ hasta obtener la masa constante, que se volvió a pesar en una balanza analítica y se expresó en $\mathrm{t} \mathrm{ha}^{-1}$.

El aporte de C de la biomasa de Canavalia se estimó por medio del secuestro o fijación del carbono ( $\left.\mathrm{C}_{\mathrm{BIOMASA}}\right)$ de la biomasa. Para ello se seleccionaron cinco plantas, se secaron según se describe en el párrafo anterior. Se utilizó la fórmula del cálculo del ( $\left.\mathrm{C}_{\text {BIOMASA }}\right)$ en especies herbáceas según el método propuesto Valera \& Carvajal, (2014).

El C promedio fijado total fue convertido a toneladas y multiplicado por la densidad absoluta total de la especie, generando acumulación de $\mathrm{C}$ por hectárea según la fórmula: $\mathrm{CA}=\mathrm{dae} * \mathrm{Ct}(1)$

Dónde: CA: C acumulado por la especie $\left(\mathrm{t} \mathrm{C} \mathrm{ha}^{-1}\right)$; dae: densidad absoluta de la especie en plantas por ha; $\mathrm{Ct}$ : $\mathrm{C}$ promedio fijado en $\mathrm{t} \mathrm{C}$ por plantas. 


\section{AMAZUNAN}

Después se determinó la relación Biomasa - $\mathrm{C}-\mathrm{CO}_{2}$ determinando la equivalencia en $\mathrm{t}$ $\mathrm{CO}_{2}$ donde a partir de la fórmula:

$\mathrm{CO}_{2}$ equiv $=\mathrm{CA} * 44$ moles $\mathrm{CO}_{2} / 12$ moles $\mathrm{C}(2)$

Dónde: $\mathrm{CO}_{2}$ equiv: toneladas por hectáreas de $\mathrm{CO}_{2} ; \mathrm{CA}$ : carbono acumulado por la especie según metodología propuesta (Valera \& Carvajal, 2014).

\section{Análisis y procedimiento estadístico}

Se comprobó la normalidad de los datos mediante la prueba de Kolmogorov-Smirnov. Se realizó el Anova simple y la significación entre medias al compararse mediante el test de Rangos Múltiples de Duncan, a $\mathbf{p} \leq 0,05$, se utilizó el STATGRAPHICS PLUS versión 5,1.

\section{Resultados y discusión}

\section{Producción de biomasa de Canavalia ensiformis y aporte de carbono de la biomasa al suelo}

La leguminosa establecida no presentó limitaciones en su crecimiento y desarrollo y mostró su potencial en rusticidad, vigorosidad y adaptabilidad. La producción de biomasa fresca total de Canavalia ensiformis cultivada en el suelo fue de $31,75 \mathrm{t} \mathrm{ha}^{-1}$ (Tabla III) y la biomasa seca total fue de 6,6 tha ${ }^{-1}$; aun cuando el experimento se repitió en la misma área los valores no fueron superados en el año siguiente, por adición no hubo efecto acumulativo.

Tabla III.

Biomasa de Canavalia ensiformis en el suelo Fluvisol Diferenciado.

\begin{tabular}{|c|c|c|c|}
\hline \multirow{2}{*}{ Tratamientos } & \multicolumn{3}{|c|}{ Biomasa fresca $\left(\right.$ tha $\left.^{-1}\right)$} \\
\hline CCs/i & Hoja & Tallo & Raíz \\
\hline CC/i & 17,62 & 9,52 & $2,49 \mathrm{c}$ \\
\hline CC/p & 23,48 & 12,50 & $2,51 \mathrm{~b}$ \\
\hline $\mathbf{X}$ & 12,33 & 13,35 & $2,61 \mathrm{a}$ \\
\hline ES $(\mathbf{x})$ & 17,43 & 11,79 & 2,53 \\
\hline & 0,655 & 0,006 & 0,004 \\
\hline
\end{tabular}

Fuente: Elaboración propia

Esta producción de biomasa fresca obtenida, se encuentra dentro del rango reportado por García et al. (2001) en Cuba en un suelo Ferralítico rojo, donde los aportes de Canavalia oscilaron entre 10 - $67 \mathrm{t} \mathrm{ha}^{-1}$ de biomasa verde. De manera similar, en el Valle del Cesar, en un Vertisol Colombia, una evaluación de cinco leguminosas (Canavalia brasiliensis, Vigna unguiculata, Clitoria ternatea, Canavalia ensiformis, Lablab purpureus) mostró la mayor producción de biomasa seca con C. ensiformis, correspondiente a 6,4 tha-1 (Castro, 2016).

En el estudio, si bien no se determinó la producción de biomasa aportada por la vegetación existente en el suelo como cobertura natural (Sorghum halepense, Amaranthus spinosus y Cyperus rotundus). Sin embargo, resultados obtenidos en un suelo Ferralítico Rojo, la cobertura natural mostró aportes de 3,6 t ha ${ }^{-1}$ de biomasa seca y 9,76 tha- de Canavalia, superando el aporte de la cobertura natural en 2,7 veces (Martín, Costa, Urquiaga \& Rivera, 2007). Por lo que existió una mayor acumulación del aporte de biomasa en los tres tratamientos donde se sembró la Canavalia respecto al testigo (barbecho), ya que esta tiene una tasa de crecimiento mucho más rápida que las arvenses. 


\section{AMAZÉNAS}

Al evaluar el efecto de la cobertura de Canavalia se estimó el secuestro o la fijación de $\mathrm{C}_{\text {BIomasa }}$ y la relación biomasa - C - $\mathrm{CO}_{2}$ (Tabla IV). Se obtuvieron valores de $3,4 \mathrm{t} \mathrm{C} \mathrm{ha}^{-1} \mathrm{de}$ Biomasa Seca (BS) total. De esta forma, el 51,5 \% de $\mathrm{C}_{\text {BIOMASA }}$ se encontró en las hojas, 39,1 \% en tallos y 9,4 \% en raíces. El carbono acumulado en la biomasa de C. ensiformis supone una captura de $\mathrm{CO}_{2}$ de la atmósfera por parte de la planta y un aporte adicional al suelo Fluvisol Diferenciado, proceso en el cual la leguminosa y el suelo participan como depósito de carbono; y permite explicar la cantidad de $\mathrm{C}$ retenido y/o secuestrado posteriormente en el suelo. Siendo la relación biomasa-C-CO $\mathrm{CO}_{2}$ estimada a partir del $\mathrm{C}_{\text {BIOMASA }}$ de $24,98 \mathrm{t} \mathrm{CO}_{2} \mathrm{ha}^{-1}$. Los resultados encontrados se enmarcan dentro de los índices normales de adsorción en el trópico, que varían de 3,2 a $10 \mathrm{t} \mathrm{C}$ por ha ${ }^{-1}$ año $^{-1}$ (Guntiñas, 2009). Es importante, considerar la estimación del C acumulado por diferentes coberturas vegetales y las emisiones de éste por el cambio del uso de las mismas y; en análisis de procesos de conversión y modificación de las coberturas en inventarios locales, regionales y nacionales (Orozco \& Lezama, 2014).

Tabla IV.

Estimación del secuestro de $C$ en la biomasa de Canavalia ensiformis y de la relación biomasa$\mathrm{C}-\mathrm{CO}_{2}$ en un suelo Fluvisol a los 80 días de sembrada.

\begin{tabular}{|c|c|c|c|}
\hline Tratamientos & $\begin{array}{c}\text { CBIOMASA }\left(\mathrm{t} \mathrm{C} \mathrm{ha}^{-1}\right) \\
\text { BS Total }\end{array}$ & $\begin{array}{c}\mathrm{C}_{\mathrm{B}} \text { integra al COS o C-COS } \\
\left(\mathrm{t} \mathrm{C} \mathrm{ha}^{-1}\right)\end{array}$ & $\begin{array}{c}\text { Biomasa - C - } \mathrm{CO}_{2} \\
\left(\mathrm{t} \mathrm{CO} \mathrm{ha}^{-1}\right)\end{array}$ \\
\hline $\mathrm{CCs} / \mathbf{i}$ & $3,15 \mathrm{~b}$ & $0,39 \mathrm{~b}$ & $23,06 \mathrm{~b}$ \\
\hline $\mathrm{CC} / \mathrm{i}$ & $4,04 \mathrm{a}$ & $0,50 \mathrm{a}$ & $29,56 \mathrm{a}$ \\
\hline $\mathbf{C C} / \mathrm{p}$ & $3,01 \mathrm{c}$ & $0,38 \mathrm{c}$ & $22,35 \mathrm{c}$ \\
\hline $\mathbf{E S}(\mathbf{x})$ & 0,011 & 0,000 & 0,019 \\
\hline
\end{tabular}

$\mathrm{C}_{\text {BIOMASA }}\left(\mathrm{t} \mathrm{C}\right.$ ha-1): aporte de $\mathrm{C}$ por la biomasa; Biomasa- $\mathrm{C}-\mathrm{CO}_{2}\left(\mathrm{t} \mathrm{CO}_{2} \mathrm{ha}^{-1}\right)$ : carbono retenido en el suelo (14). Fuente: Elaboración propia.

Del mismo modo se ha observado en algunos estudios, contenidos promedios de $\mathrm{C}$ en plantas herbáceas de 50,98 \% de BS y en Canavalia 50,15\%, valores similares al estimado de 51,5\% de $\mathrm{C}$ obtenido en BS de esta leguminosa en el estudio a los 80 días de sembrada. Igualmente, otro estudio demostró que el C orgánico en el suelo aumentó en un $22 \%$ con el empleo de Canavalia ensiformis (Castro, 2016). Otros autores consideran necesario identificar las mejores prácticas de manejo por agroecosistemas que conlleven a la estabilización del C en el suelo (García et al, 2015). El carbono determinado no solo permitió conocer el nivel de adsorción del C por el tipo de cubierta; sino evaluar la cantidad retenida en el suelo y la repercusión sobre las propiedades químicas analizadas.

\section{Efecto del uso de Canavalia ensiformis en algunas propiedades químicas del suelo.}

Se valoró, incrementos en los niveles de carbono orgánico del suelo (CO) y el carbono orgánico total (COT); fundamentalmente donde se incorporó la leguminosa o permaneció como cobertura permanente. Sin embargo, aunque los contenidos de CO y COT no son elevados, establecen una diferencia con relación al suelo en estado inicial. Ello evidenció que el contenido de $\mathrm{C}$ acumulado en la biomasa de la Canavalia (Tabla IV) incidió sobre el contenido posterior y la ganancia de este elemento en el suelo (Tabla V).

Al evaluar el comportamiento del carbono orgánico (CO) en el tratamiento en barbecho (Cn) a (0 días) y 120 días mostró diferencias, lo que sugiere que los aportes de biomasa de la cobertura natural fueron suficientes para estimular un cambio en el contenido de $\mathrm{C}$ en el suelo a los 120 días. 
Y aunque las plantas establecidas en barbecho fijan C, no alcanzan la tasa de crecimiento y desarrollo comparado con la Canavalia. De ahí, se atribuya un aporte de $\mathrm{C}$ al suelo desde las especies presentes en el suelo en condiciones de barbecho (Sarandón \& Flores, 2014). Aun cuando el experimento se repitió en la misma área no hubo adición por efecto acumulativo, siendo pobre y poco persistente la adición de carbono en el suelo comparado con el resto de los tratamientos. En el tratamiento Canavalia sin incorporar (CCs/i) no hubo grandes modificaciones en el contenido de $\mathrm{CO}$ en el suelo a los 120 días con relación al momento 0 días, pero sí mejoró el contenido del COT. Las explicaciones pudieran estar en la cobertura de la leguminosa sobre la superficie del suelo aun cuando realiza una contribución del elemento al suelo no lo hizo en la misma magnitud que el resto de los tratamientos. La descomposición y transformación de los residuos de está solo se hace efectiva cuando entran en contacto directo con la superficie del suelo y los organismos que en el habitan y; a la baja relación C: N. Siendo probable y normal que, al ser expuesta la biomasa, parte del $\mathrm{C}$ fijado en ella se pierda por volatilización a la atmósfera en forma de $\mathrm{CO}_{2}$.

Tabla V.

Comportamiento del carbono orgánico y carbono orgánico total en el suelo Fluvisol Diferenciado con C. ensiformis a los 0 y 120 días.

\begin{tabular}{|c|c|c|c|}
\hline Momento & Tratamiento & CO $(\%)$ & COT $\left(\mathrm{t} \mathrm{ha}^{-1}\right)$ \\
\hline \multirow{3}{*}{$\mathbf{0}$ días } & $\mathrm{Cn}$ & $1,0 \mathrm{~d}$ & $25,2 \mathrm{e}$ \\
\hline \multirow{3}{*}{$\mathbf{1 2 0}$ días } & $\mathrm{Cn}$ & $1,12 \mathrm{c}$ & $27,7 \mathrm{~d}$ \\
\cline { 2 - 4 } & $\mathrm{CC} / \mathrm{i}$ & $1,16 \mathrm{c}$ & $28,0 \mathrm{c}$ \\
\cline { 2 - 4 } & $\mathrm{CC} / \mathrm{i}$ & $1,28 \mathrm{~b}$ & $30,2 \mathrm{~b}$ \\
\cline { 2 - 4 } & $\mathrm{CC} / \mathrm{p}$ & $1,42 \mathbf{a}$ & $32,9 \mathbf{a}$ \\
\cline { 2 - 4 } & $\mathrm{ES}(\mathrm{x})$ & 0,023 & 0,022 \\
\hline
\end{tabular}

Medias con letras diferentes en la misma columna difieren entre sí según Prueba de Duncan para $p \leq 0,05$. Leyenda: $\mathrm{CO}(\%)$ según Walkley-Black; COT $\left(\mathrm{t} \mathrm{ha}^{-1}\right)=\mathrm{CO} \times \mathrm{Da} \times \mathrm{P}(12)$. Cn: Cobertura natural, CCs/i: Cobertura de Canavalia sin incorporar, CC/i: Cobertura de Canavalia incorporada, $\mathrm{CC} / \mathrm{p}$ : Cobertura de Canavalia permanente.

Fuente: Renté, (2019).

Por otra parte, los tratamientos $\mathrm{CC} / \mathrm{i}$ y $\mathrm{CC} / \mathrm{p}$ incrementan los valores de $\mathrm{CO}$ y $\mathrm{COT}$, siendo superiores con la cobertura permanente. Es indudable que el $\mathrm{C}$ que acumulado en la biomasa de la Canavalia, beneficio la alta tasa de captura y fijación de $\mathrm{C}$ y $\mathrm{N}$, e incidió sobre la composición e incremento posterior en el suelo (Tabla V). Los resultados superiores hallados en la CC/p de manera particular, demuestran que cuando el ciclo biológico de la leguminosa no se interrumpe, se favorece la captura y fijación biológica de $\mathrm{C}$ y N. Entonces, se obtiene mayor producción de biomasa, secreción de exudados radiculares que aportan este y otros elementos que benefician el proceso de humificación. Aunque el contenido de COT se incrementó con la leguminosa (en el orden de $30 \mathrm{t} \mathrm{ha}^{-1}$ ), estos valores se encuentran por debajo de la media del COT de algunos suelos del país, tal es el caso de los Ferralíticos Rojos con valores de $32 \mathrm{t} \mathrm{ha}^{-1}$ (Ríos, Vargas \& Funes, 2011).

La tabla VI., muestra las variables químicas analizadas; el pH en el suelo se mantuvo en el rango de mayor disponibilidad y aprovechamiento de nutrientes. El contenido de $\mathrm{N}$ total, $\mathrm{P}_{2} \mathrm{O}_{5}$, $\mathrm{Ca}^{(2+)}$ y $\mathrm{Mg}^{(2+)}$, incrementaron cuando se estableció la Canavalia en sus diferentes variantes. En todos los casos los nutrientes incrementaron su contenido cuando la leguminosa fue incorporada al suelo o se mantuvo permanentemente. 
Tabla VI.

Comportamiento de propiedades químicas del suelo Fluvisol Diferenciado ante diferentes manejos de Canavalia ensiformis, a los 120 días.

\begin{tabular}{|c|c|c|c|c|c|c|c|c|}
\hline \multicolumn{2}{|c|}{ Tratamiento } & \multirow{2}{*}{$\begin{array}{c}\mathrm{pH} \\
(\mathrm{KCl})\end{array}$} & \multirow[t]{2}{*}{$\mathbf{N}$ total $(\%)$} & \multirow{2}{*}{\multicolumn{2}{|c|}{$\begin{array}{l}\mathrm{P}_{2} \mathrm{O}_{5} \\
\left(\mathbf{m g 1 0 0} \mathbf{g}_{2}^{-1} \mathrm{O}\right.\end{array}$}} & $\mathrm{Ca}^{(2+)}$ & $\mathrm{Mg}^{(2+)}$ & $\mathrm{Na}^{(+)}$ \\
\hline & & & & & & \multicolumn{3}{|c|}{$\left(\mathrm{cmol}_{\mathrm{c}} \mathrm{kg}^{-1}\right)$} \\
\hline 0 días & Cn & 6,7 & $0,097 \mathrm{e}$ & $199,8 \mathrm{e}$ & 42,52 & $16,50 \mathrm{~b}$ & $4,50 \mathrm{c}$ & 0,46 \\
\hline \multirow[t]{5}{*}{120 días } & Cn & 6,7 & $0,106 \mathrm{c}$ & $206,4 \mathrm{c}$ & 48,86 & $11,66 \mathrm{e}$ & $4,48 \mathrm{c}$ & 0,27 \\
\hline & $\mathbf{C C s} / \mathbf{i}$ & 6,7 & $0,100 \mathrm{~d}$ & $201,3 \mathrm{~d}$ & 44,85 & $14,15 \mathrm{c}$ & $3,27 \mathrm{~d}$ & 0,23 \\
\hline & $\mathbf{C C} / \mathbf{i}$ & 6,7 & $0,121 \mathrm{~b}$ & $211,0 \mathrm{~b}$ & 47,52 & $13,18 \mathrm{~d}$ & $5,91 \mathrm{~b}$ & 0,22 \\
\hline & $\mathbf{C C} / \mathbf{p}$ & 6,3 & $0,135 \mathbf{a}$ & $213,4 \mathbf{a}$ & 46,13 & $18,85 \mathbf{a}$ & $9,54 \mathbf{a}$ & 0,39 \\
\hline & $\mathbf{E S}(\mathbf{x})$ & Ns. & 0,0005 & 0,2305 & Ns. & 0,0215 & 0,0228 & Ns. \\
\hline
\end{tabular}

Medias con letras diferentes en la misma columna difieren entre sí según prueba de Duncan para $p \leq 0,05$. Leyenda: $\mathrm{Cn}$ : Testigo. Cobertura natural, CCs/i: Cobertura de Canavalia sin Incorporar, CC/i: Cobertura de Canavalia Incorporada, CC/p: Cobertura de Canavalia Permanente, Ns: no significativo. Fuente: Elaboración propia.

El contenido de $\mathrm{N}$ total entre los tratamientos mostró diferencias, resultando $\mathrm{CC} / \mathrm{i}$ y CC/p los de mayores valores, con un comportamiento similar al COT (Tabla V). La mineralización del $\mathrm{N}$ en el suelo depende en gran medida de la relación C: $\mathrm{N}$ del material incorporado. Los resultados de este estudio sugieren que la leguminosa por su baja relación C: $\mathrm{N}$ realizó una conversión y descomposición rápida del material vegetal, tal como explican Martín \& Rivera (2002). También refieren diversos autores resultados similares al emplear Canavalia como AV/CC, los que atribuyen un efecto marcado en la fijación de nitrógeno y en el aporte de materia orgánica (Prager et al, 2012).

El contenido de $\mathrm{P}$ en el suelo fue alto desde el inicio del estudio, lo que puede atribuirse a los procesos de formación y los materiales primarios ricos en $\mathrm{P}$ en este suelo, así como a posibles aplicaciones continuadas de portadores de este nutriente y acumuladas en él. Pudiera ser esto posible por la poca movilidad del $\mathrm{P}$ en el suelo, que facilita la acumulación en las capas superficiales. No obstante, se encontraron diferencias significativas entre los tratamientos, siendo los contenidos de este elemento mayores en los tratamientos cuando la leguminosa se incorporó (CC/i) o se mantuvo permanente $(\mathrm{CC} / \mathrm{p})$ al coincidir con lo reportado que estas plantas que mejoran el ciclo del elemento (Sánchez et al, 2010).

Los contenidos de K y Na no mostraron diferencias entre los tratamientos. Determinados abonos verdes en condiciones de espacio y temporada tienden favorecer la capacidad de promover variaciones de producción dentro de una misma especie. Por ello factores como la época de siembra o el ambiente (luminosidad, temperatura, entre otras) interfieren en la producción vegetativa en cuanto a la concentración de nutrientes de las leguminosas factores (Aparecida et al, 2000). También pudiera atribuirse a algún desbalance con otros nutrientes, limitando la movilidad del potasio en el suelo.

Los contenidos de los cationes $\mathrm{Ca}$ y $\mathrm{Mg}$ mostraron incrementos en los tratamientos con Canavalia y los mejores resultados se obtuvieron con el establecimiento de forma permanente de esta leguminosa, atribuido a la capacidad de retornar el Ca y el Mg a través de su biomasa. Los efectos que producen los abonos verdes en las propiedades químicas del suelo son variables y dependen de factores como la especie utilizada, el manejo de la biomasa, la época de plantación, el corte del abono, el tiempo de permanencia de los residuos en el suelo y de las condiciones locales, así como de la interacción de todos esos factores (Aparecida et al., 2000). Y podría añadirse a las condiciones del suelo. 


\section{Conclusiones}

La Canavalia ensiformis como cultivo de cobertura constituye una alternativa nutricional promisoria, al mejorar la condición natural del suelo Fluvisol. Elconsenso del manejo de esta leguminosa al agroecosistema es que permite aportar carbono por concepto de biomasa, y mejora las propiedades químicas. El cultivo de cobertura mejora el suelo en estudio cuando es incorporada o mantenida permanentemente en el suelo.

\section{Referentes Bibliográficas}

Anaya, A. \& Huber, S.E. (2015). Long - term soil organic carbon and nitrogen dynamics after conversion of tropical forest to traditional sugar cane agriculture in East México. Soil Tillage Res. 147, p.20-29.

Aparecida, F., Fortini A. E, Bueno, M., Adelante, H. \& Muriz, J.A. (2000). Adubação na recupeção da fertilidade de un Latossolo vermelho - escuro degradado. Pesq. Agropec. Bras., Brasilia, v.35 (2), p 277-288, Fev 2000.

Beuchelt, T. D., Camacho, V.C.T., GöhringL, Hernández, R.V.M., Hellin., Sonder, K., et al. (2015). Social and income trade-offs of conservation agriculture practices on crop residue use in Mexico's central highlands. Agric. Syst. 134, p.61-75.

Bojórquez, S.J., Castillo, P.L.A., Hernández, J.A., García, P.J.D. \& Madueño, M.A. (2015). Cambios en las reservas de carbono orgánico del suelo bajo diferentes coberturas. Cultivos Tropicales, 36(4): 63-69. ISSN impreso: 0258-5936, ISSN digital: 1819 - 4087.

Bunch, R. (2016). ¿Cómo prohibir las sequías? Aprendiendo de los africanos que ya aprendieron de los latinoamericanos. Revista de Agroecología. LEISA. 32(2), p 8 -11. ISSN 1729 7419.

Carvalho, J.E.B., Dias, R.C. dos S. \& Melo Filho J.F. (2006). Produção integrada x convencional - impacto sobre a qualidade do solo. Cruz das Almas: Embrapa Mandioca e Fruticultura Tropical, $4 \mathrm{p}$.

Castro, E. (2016). Utilización de leguminosas forrajeras como abonos verdes para la producción de cultivos forrajeros y leche en ganaderías doble propósito en el trópico seco. (Tesis de Doctorado). Universidad Nacional de Colombia. Revista de la Facultad de Medicina Veterinaria y Zootecnia. DOI: 10.15446/rfmvz.

Dendooven, L, Gutiérrez, OVF, Patiño, ZL, Ramírez, VDA, Verhulst, N, Luna, G M, et al. (2012). Greenhouse gas emissions under conservation agriculture compared to traditional cultivation of maize in the central highlands of México. Sci. Total, Environ. 43, p 23744.

Embrapa, Agrobiología. Base de dados. Leguminosas. (2007). [cited 2015, 2016 diciembre]. Available from: http://intranet2.cnpab.EMBRAPA.br/leguminosas/ detalhesbusca.asp?Cod id=12\&tema=resumo.

García, F.N., Albaladejo, J., Almagro, M. \& Martínez, M. (2015). Beneficial effects of reduced tillage and green manure on soil aggregation and stabilization of organic carbon in a Mediterranean agroecosystem. Soil Tillage Res. 153, p.66 -75.

García, M., Treto, E. \& Mayté Alvarez. (2001). Comportamiento de diferentes especies de plantas para ser utilizadas como abonos verdes en las condiciones de Cuba. Revista Cultivos Tropicales, vol. 22, no. 4, p. 11-16. PDF.

García, R.M., Rivera E.R., Cruz H.Y., Acosta A.Y. \& Ramón C.J. (2017). Respuesta de Canavalia ensiformis (L.) a la inoculación con diferentes cepas de hongo micorrízico arbuscular en un suelo Ferralítico Amarillo Rojizo Lixiviado. Cultivos Tropicales. Vol. 38(1): p 7-12. ISSN impreso: 0258-5936 ISSN digital: 1819-4087.

Guntiñas, R. Ma. E. (2009). Influencia de la temperatura y de la humedad en la dinámica de la materia orgánica de los suelos de Galicia y su relación con el cambio climático. (Tesis de Doctorado). Instituto de Investigaciones Agrobiológicas de Galicia. Universidad de Santiago de Compostela. España, p. 749. 


\section{AMAZÉNAS}

Hernández, A., Pérez, J., Bosch, D. \& Castro, N. (2015). Clasificación de los suelos de Cuba. Mayabeque, Cuba. Ediciones INCA. p. 93. ISBN 978-959-7023-77-7.

INIA, Instituto Nacional de Investigación Agropecuaria. (2015). Secuestro de carbono. Jornada de Puertas Abiertas - INIA. Tacuarembó. [cited 2015 May 5]. p. 15 -17. Available from: www.inia.uy.

INIA. Instituto Nacional de Investigación Agropecuaria. (2008). Jornada Abonos verdes: De Invierno. Uruguay. Serie Actividades de Difusión No.546. Programa de Difusión Nacional. Jornada de Puertas Abiertas-INIA. [cited 2014 febrero 2]. Available from: www.inia.uy.

Jiménez, M. A. Ma., Reyes, S.J.T. \& Silveira, Ma. L. (2011). Secuestro y Distribución de Carbono Orgánico del Suelo Bajo Diferentes Sistemas de Manejo de Pasturas. University of Florida, IFAS [cited May 5]. Available from: http://edis.ifas.ufl.edu.

Martín G. M., Rivera R., Arias L. \& Pérez A. (2012). Respuesta de la Canavalia ensiformis a la inoculación micorrízica con Glomus cubense (cepa INCAM-4), su efecto de permanencia en el cultivo del maíz. Cultivos Tropicales, vol. 33 (2), p. 20 -28, ISSN 0258-5936. 9.

Martín G.M. \& Rivera R. (2002). Participación del nitrógeno de los abonos verdes en la nutrición nitrogenada del Maíz (Zea mays L.) cultivado sobre suelo Ferralítico rojo. Cultivos Tropicales. Vol. 23 (3), p. 91-96.

Martín, G. M, Costa, R. J., Urquiaga, S. \& Rivera, R.A. (2007). Rotación del Abono Verde Canavalia ensiformis (L.) con maíz y micorrizas. Cultivos Tropicales. 57(4), p 313 - 21.

Nahed, J., Valdivieso, P. A., Aguilar, J., Cámara, C.J. \& Grande, C. (2013). Silvopastoral systems with traditional management in southeastern Mexico: prototype of livestock agroforestry for cleaner production. J. Cleaner Prod. 57, p 266.

Orozco, H.M.E. \& Lezama, M.P. (2014). Carbono en ambientes biofísicos y productivos línea base sobre un cambio climático.1st ed. Toluca, Mexico: Ediciones Instituto Literario 100 Ote. [cited 2014]. Available from: http://www. uaemex.mx/ISBN: 978-607-422503-7.

Paneque, P.M., Calaña, N.J.M., Calderón, V.M., Borges, B.Y., Hernández, G.T.C., Caruncho, C.M. (2010). Manual de técnicas analíticas para análisis de suelo, foliar, abonos orgánicos y fertilizantes químicos 1st ed. Mayabeque, Cuba: Ediciones INCA. [cited 2016 Marz 3]. Available from: http://mst.ama.cu/578/. p.157.

Prager, M., Sanclemente, O., Sánchez, de P. M., Gallego, J. \& Ángel, D. (2012). Abonos verdes: Tecnología para el manejo agroecológico de los cultivos. En Revista Agroecología, Murcia España. No. 7, p.53 - 62.

Renté, O. (2019). Canavalia ensiformis (L): In the contribution of organic carbon to Eutric Differentiated soils. American Journal of Applied Scientific Research. 5 (2): 41-46. http://www.sciencepublishinggroup.com/j/ajasr.doi:10.11648/j.ajasr.2019 050212.ISSN: 2471-97 22 (Print); ISSN: 2471-9730 (Online).

Riofrío, G. (2007). Cuantificación del carbono almacenado en agroforestales en la estación experimental Santa Catalina, INIAP, Ecuador. Tesis de Licenciatura. Escuela Superior Politécnica de Chimborazo, Riobamba, Ecuador. (cited 2015 May 5). Available from: http://www.esscribd.com/doc/35448943/Cuantificacion-de-carbonoensistemasagroforestales.

Ríos, L.H., Vargas, B.D. \& Funes, M.F. (2011). Innovación agroecológica, adaptación y mitigación del cambio climático. 1st ed. Mayabeque, Cuba: Ediciones INCA. ISBN 9789597023-53-1.

Sánchez de, P. M., Posada, R., Velásquez, D. \& Narváez, M. (2010). Metodologías básicas para el trabajo con Micorriza arbuscular y hongos formadores de micorriza arbuscular. Universidad Nacional de Colombia, Palmira.

Sarandón, S.J. \& Flores, C.C. (2014). Agroecología: bases teóricas para el diseño y manejo de Agroecosistemas sustentables. Facultad de Ciencias Agrarias y Forestales. 1a ed. La Plata: Universidad Nacional de La Plata. E-Book: ISBN 978-950-34-1107-0.

Sociedad de agricultores de Chile. (2013). Boletín de Agricultura y abonos verdes. [cited 2015 Marz 3]. Available from: http://www.Abcagro.Com/fertilizantes/abonos verdes.asp. 
Valera, V. \& Carvajal, N. (2014). Valoración económica del secuestro de carbono en un sector del Parque Nacional Waraira Repano (Caracas, Venezuela). ANALES Universidad Metropolitana. Universidad Simón Bolívar. 14(2), 241-64.

Walkley, A. \& Black, I.A. (1934). An examination of the Degtjareff method for determining soil organic matter, and a proposed modification of the chromic acid titration method. Soil science, Vol. 37(1) p. 29 - 38. ISSN 0038-075X, 1538-9243. 